Invited talk, $6^{\text {th }}$ Conference on the Intersections of Particle and Nuclear Physics, Big Sky, 1997

\title{
Pentaquark Phenomenology
}

\author{
Harry J. Lipkin ${ }^{a, b, c 1}$ \\ Alis 221997 \\ a Department of Particle Physics Weizmann Institute of Science, Rehovot 76100, Istael \\ USTI \\ b School of Physics and Astronomy, Raymond and Beverly Sackler Faculty of Exact \\ Sciences, Tel Aviv University, Tel Aviv, Israel \\ ' High Energy Physics Division, Argonne National Laboratory, Argonne, IL 60439-4815, \\ USA
}

TECERED

\begin{abstract}
Heavy flavor hadron scattering and bound states give experimental informution otherwise unobtainable on effective $(q q)_{6}$ and $(\bar{q} q)_{8}$ interactions. All constitu nut quark model successes in (uds) hadron spectroscopy depend only on $(q q)_{3 .}$ and $(\bar{q} q)_{1}$ interactions. Reliable vertex detectors open new pentaquark search directions. Any event in with a proton emitted from a secondary vertex indicates a particle decaying weakly by proton emission and the discovery of a new particle if its mass is higher than that of known charmed baryons. There is no combinatorial background and striking decay signatures like $p \phi \pi^{-}$are no longer needed.
\end{abstract}

\section{INTRODUCTION - THE BLIND MEN AND THE ELEPHANT}

Why look for an anticharmed strange baryon? Who cares whether it is bound? Answer: It can help understanding how QCD makes hadrons [1] QCD does not yet explain everything. Present attempts to describe hadrons recall the story of the blind men and the elephant.

- A pion is a Goldstone Boson - A proton is a Skyrmion

- A pion is two-thirds of a proton. The simple quark model prediction $\sigma_{\text {toot }}\left(\pi^{-} p\right) \approx(2 / 3) \cdot \sigma_{\text {tot }}(p p)[2,3]$ still fits experimental data better than $7 \%$ up to $310 \mathrm{Gev} / \mathrm{c}[4]$.

1) Supported in part by grant No. I-0304-120-.07/93 from The German-Israeli Foundation for Scientific Research and Development and by the U.S. Department of Energy, Division of High Energy Physics, Contract W-31-109-ENG-38. 


\section{DISCLAIMER}

This report was prepared as an account of work sponsored by an agency of the United States Government. Neither the United States Government nor any agency thereof, nor any of their employees, makes any warranty, express or implied, or assumes any legal liability or responsibility for the accuracy, completeness, or usefulness of any information, apparatus, product, or process disclosed, or represents that its use would not infringe privately owned rights. Reference herein to any specific commercial product, process, or service by trade name, trademark, manufacturer, or otherwise does not necessarily constitute or imply its endorsement, recommendation, or favoring by the United States Government or any agency thereof. The views and opinions of authors expressed herein do not necessarily state or reflect those of the United States Government or any agency thereof. 


\section{DISCLAMIER}

Portions of this docoment moy be illegible in electronic image products. Images are produced from the best available original docroment. 
- Mesons and Baryons are made of the same quarks. Describing both as simple composites of asymptotically free quasiparticles with a unique effective mass value predicts hadron masses, magnetic moments and hyperfine splittings [5-7].

- Lattice QCD can give all the answers.

- Lattice calculations disagree on whether the H dibaryon is bound. No hope of settling this question until much bigger lattices are available.

- Light (uds) SU(3) symmetry and Heavy Quark symmetry (cbt) are good

- Light (uds) SU(3) symmetry is bad. All nontrivial hadron states violate $\mathrm{SU}(3)$. All light $\mathrm{V}, \mathrm{A}$ and $\mathrm{T}$ mesons have good isospin symmetry with flavor mixing in (u.d) space and no $s \bar{s}$ component; e.g. $\rho, \omega$.

- The s-quark is a heavy quark. Flavor mixing in mass eigenstates predicted by SU(3) is not there. Most nontrivial strange hadron states satisfy ( $\mathrm{scb}$ ) heavy quark symmetry with no flavor mixing.; e.g. $\phi, \psi, \Upsilon$.

An underlying dynamics describes many meson and baryon properties primarily in terms constituent quarks which are the same in mesons and baryons.

$$
\begin{aligned}
& \left\langle m_{s}-m_{u}\right\rangle_{B a r}=M_{\Lambda}-M_{N}=177 \mathrm{MeV}= \\
= & \frac{M_{N}+M_{\Delta}}{6} \cdot\left(\frac{M_{\Delta}-M_{N}}{M_{\Sigma^{*}}-M_{\Sigma}}-1\right)=190 \mathrm{MeV} . \\
\left\langle m_{s}-\right. & \left.m_{u}\right\rangle_{\text {mes }}=\frac{3\left(M_{K^{*}}-M_{\rho}\right)+M_{K}-M_{\pi}}{4}=180 \mathrm{MeV}= \\
= & \frac{3 M_{\rho}+M_{\pi}}{8} \cdot\left(\frac{M_{\rho}-M_{\pi}}{M_{K^{*}}-M_{K}}-1\right)=178 \mathrm{MeV} .
\end{aligned}
$$

The mass difference $m_{s}-m_{u}$ has the same value $\pm 3 \%$ when calculated in two independent ways from baryon masses and meson masses [5-7]. The same approach applied to $m_{b}-m_{c}$ gives

$$
\begin{gathered}
\left\langle m_{b}-m_{c}\right\rangle_{B a r}=M\left(\Lambda_{b}\right)-M\left(\Lambda_{c}\right)=3356 \mathrm{MeV} \\
\left\langle m_{b}-m_{c}\right\rangle_{m e s}=\frac{3\left(M_{B^{*}}-M_{D^{*}}\right)+M_{B}-M_{D}}{4}=3338 \mathrm{MeV} .
\end{gathered}
$$

The ratio $\frac{m_{e}}{m_{*}}$ has the same value $\pm 2.5 \%$ for mesons and baryons.

$$
\left(\frac{m_{s}}{m_{u}}\right)_{B a r}=\frac{M_{\Delta}-M_{N}}{M_{\Sigma^{*}}-M_{\Sigma}}=1.53=\left(\frac{m_{s}}{m_{u}}\right)_{M e s}=\frac{M_{\rho}-M_{\pi}}{M_{K^{*}}-M_{K}}=1.61
$$

Three magnetic moment predictions with no free parameters $[8,9]$

$$
\mu_{\Lambda}=-0.61 \mathrm{n} . \mathrm{m} .=-\frac{\mu_{p}}{3} \cdot \frac{m_{u}}{m_{s}}=-\frac{\mu_{p}}{3} \frac{M_{\Sigma^{*}}-M_{\Sigma}}{M_{\Delta}-M_{N}}=-0.61 \mathrm{n} . \mathrm{m} .
$$




$$
\begin{gathered}
-1.46=\frac{\mu_{p}}{\mu_{n}}=-\frac{3}{2} \\
\mu_{p}+\mu_{n}=0.88 \mathrm{n} . \mathrm{m} .=\frac{M_{p}}{3 m_{u}}=\frac{2 M_{p}}{M_{N}+M_{\Delta}}=0.865 \mathrm{n} . \mathrm{m} .
\end{gathered}
$$

QCD calculations have not yet explained such remarkably successful simple constituent quark model results. A search for new experimental input to guide us is therefore of interest.

\section{EXOTIC HADRONS AND HEAVY FLAVORS - A WINDOW INTO QCD}

Additional input comes from two striking features of the hadron spectrum:

- Absence of strongly bound multiquark exotic states like a dipion with a mass less than two pion masses or a dibaryon bound by $100 \mathrm{MeV}$.

- Nuclear structure described by three-quark clusters called nucleons.

The constituent quark model gives a very simple answer [10]. The one gluon exchange ansatz for $\frac{V(q \bar{q})_{s}}{V(q \bar{q})_{1}}$ and $\frac{V(q q)_{6}}{V(q q)_{3 *} *}$ gives:

- Color-exchange color-electric interaction saturates [11] - no forces between color singlet hadrons.

- Color electric energy unchanged by color recoupling.

- Color magnetic $q q$ forces repulsive for a single flavor - attractive between s quark in $D_{s}$ or $B_{s}$ and $u$ and $d$ quarks in proton.

- Energy gain by color-spin recoupling can bind $H$ (hexaquark) [12], charmed and beauty pentaquarks $[1,13-18] P_{c}=\bar{c} s u u d ; P_{b}=\bar{b} s u u d$.

The validity of this simple picture still remains to be confirmed by experiment since no experimental information is yet available about short-range color-sextet or color-octet two-body interactions. All constituent quark model successes with a two-body color-exchange interaction $[6-8,11]$ and all hadron spectroscopy without exotics including scattering depend only upon $(\bar{q} q)_{1}$ and $(q q)_{3 *}$ interactions Baryon-nucleon scattering in the $(\mathrm{u}, \mathrm{d}, \mathrm{s})$ sector is dominated by a short-range color-magnetic repulsion (well-known repulsive core in the nucleon-nucleon interaction). Meson-hadron scattering in the (uds) sector must have either a quark or an antiquark in the meson with the same flavor as a quarks in the other hadron. A $q q$ pair of the same flavor has a repulsive color-magnetic interaction keeping the two hadrons apart. A $\bar{q} q$ pair of the same flavor can annihilate and produce a hadron resonance. Thus hadronhadron scattering in the $(\mathrm{u}, \mathrm{d}, \mathrm{s})$ sector is dominated either by $q q$ repulsion or by resonances produced by $\bar{q} q$ annihilation. 
Only with more than three flavors can the $(q q)_{6}$ or $(\bar{q} q)_{s}$ interactions be observed in realistic scattering experiments (unrealistic cases like $K^{-} \Delta^{-}$and $\phi N$ are excluded) with no common flavor between beam and target. Thus the possible existence of exotic hadrons remains crucial to understanding how QCD makes hadrons from quarks and gluons [19].

The $H$ dibaryon [12] was shown to have a gain in color-magnetic energy over the $\Lambda \Lambda$ system $[12,20]$. But a lattice calculation [21] showed a repulsive $\Lambda-\Lambda$ interaction generated by quark exchange $[22,23]$ not included in simple model calculations which could well prevent the six quarks from coming close enough together to feel the additional binding of the short range color-magnetic interaction. Pentaquarks, shown [13-18] to have a color-magnetic binding roughly equal to the $H$, have no possible quark exchange force in the lowest decay channel $D_{s} N[22]$. The simplest lattice calculation can easily be done in parallel with the more complicated $\mathrm{H}$ calculation both in the symmetry limit where all light quarks have the same mass and with $S U(3)$ symmetry breaking. Comparing results may provide considerable insight into the physics of QCD in multiquark systems even if the pentaquark is not bound. However, no such lattice calculation has been done or is planned.

\section{GOOD VFRTEX DETECTORS CREATE NEW BALL GAME}

Without vertex detectors a pentaquark decay signal appears against a large combinatorial background. Peaks in a mass spectrum can arise from statistical fluctuations in the background. Standard statistical considerations and good signatures like $p \phi \pi^{-}$are needed to analyze data.

Good vertex detectors eliminate all combinatorial background. An event with a decay proton from a secondary vertex cannot be a statistical fluctuation of known physics. If its mass differs from that of known weakly decaying baryons, it indicates a new as yet unknown particle. Searches for such secondary-vertex protons are open searches for new weakly decaying baryons and might even find new physics beyond the standard model. This point has not been noted in previous articles on pentaquark searches $[24,28]$

A weakly-decaying baryon should produce not only a peak in the mass spectrum, but also a tail below corresponding to decays where neutral particles have escaped detection. Events containing a muon or electron are expected and particularly significant since weak decays via a $W$ always have semileptonic modes.

The original suggestion [13] directing the search to striking signatures like $p \phi \pi^{-}$no longer holds with good vertex detectors. One might better begin with an extremely stringent cut on the proton to select all events with protons really from a secondary vertex. Even and odd prong events correspond respectively to decays of neutral and charged particles. A charged pentaquark with the 
structure of a $D$, bound to a neutron rather than a proton would be more apt to decay to a final state containing a neutron rather than a proton, unless the final state baryon is a $\Delta^{\circ}$ or $N^{* 0}$ decaying to $p \pi^{-}$. This immediately suggests looking for $p \pi^{-}$resonances in all odd prong events.

Quasi-two-body events with protons definitely coming from a secondary vertex have unique energies providing a striking signal. Selection of a proton with an energy near the appropriate value would enhance signal/noise ratios by a considerable factor in modes like $p \pi^{-}$decay which would be impossible signatures without vertex detectors.

The particle-ID and vertex detector in the Fermilab experiment [27] are not sufficiently reliable to identify events as definitely arising from secondary protons. Their small number of events do not provide sufficient evidence for a new particle, but are not easily dismissed as due to known systematics. Better experiments with reliable vertex detectors are needed to resolve this question.

\section{ACKNOWLEDGEMENT}

Many stimulating discussions with the Tel Aviv experimental group [25,28] are gratefully acknowledged and in particular Danny Ashery, Sharon May-Tal Beck, Gilad Hurvits, Jechiel Lichtenstadt and Murray Moinester.

\section{REFERENCES}

1. Harry J. Lipkin, Nucl. Phys. A, in press and references therein

2. E. M. Levin and L. L. Frankfurt, Zh. Eksperim. i. Theor. Fiz.-Pis'ma Redakt (1965) 105; JETP Letters (1965) 65

3. H.J. Lipkin and F. Scheck, Phys. Rev. Lett. 16 (1966) 71

4. Harry J. Lipkin, Physics Letters B335 (1994) 500

5. Ya. B. Zeldovich and A.D. Sakharov, Yad. Fiz 4 (1966)395; Sov. J. Nucl. Phys. 4 (1967) 283

6. I. Cohen and H. J. Lipkin, Phys. Lett. 93B, (1980) 56

7. Harry J. Lipkin, Phys. Lett. B233 (1989) 446; Nuc. Phys. A507 (1990) 205c

8. A. De Rujula, H. Georgi and S.L. Glashow, Phys. Rev. D12 (1975) 147

9. Harry J. Lipkin, Nucl. Phys. A478, (1988) 307c

10. H.J. Lipkin, Phys. Lett. 198B (1987) 131

11. H.J. Lipkin, Phys. Lett. 45B (1973) 267

12. R. L. Jaffe, Phys. Rev. Lett. 38, (1977) 195

13. Harry J. Lipkin, in Hadrons, Quarks and Gluons, Proceedings of the Hadronic Session of the XXIInd Rencontre de Moriond, Edited by J. Tran Thanh Van, Editions Frontieres, Gif Sur Yvette - France (1987), p.691

14. Harry J. Lipkin, In The Elementary Structure of Matter, Proceedings of the Workshop, Les Houches, France, 1987 Edited by J.-M. Richard et al, SpringerVerlag (1987) p.24 
15. Harry J. Lipkin, in Hadron '87, Proceedings of the Second International Conference on Hadron Spectroscopy, KEK Tsukuba, Japan, edited by Y. Oyanagi, K. Takamatsu and T.Tsuru, KEK Report 87-7 (1987), p.363.

16. Harry J. Lipkin, In Proceedings of PANIC '87, XI International Conference on Particles and Nuclei, Nucl. Phys. A478, 307c (1988)

17. Harry J. Lipkin, Phys. Lett. 195B, (1987) 484

18. C. Gignoux, B. Silvestre-Brac and J. M. Richard, In The Elementary Structure of Matter, Proceedings of the Workshop, Les Houches, France, 1987 Edited by J.-M. Richard et al, Springer-Verlag (1987) p.42; Phys. Lett. B193 (1987) 323

19. Harry J. Lipkin, In Intersections Between Particle and Nuclear Physics, Proc. Conf. on The Intersections Between Particle and Nuclear Physics, Lake Louise, Canada, 1986 Edited by Donald F. Geesaman AIP Conference Proceedings No. 150, p. 657

20. J. L. Rosner, Phys. Rev. D 33 (1986) 2043

21. P. MacKenzie and H. Thacker, Phys. Rev. Letters 65, 2539 (1985)

22. Harry J. Lipkin, In Proceedings of the International Symposium on The Production and Decay of Heavy Flavors, Stanford (1987) Edited by Elliott.D. Bloom and Alfred Fridman, Annals of the New York Academy of Sciences, Vol. 535 (1988) p.438

23. H. Thacker, private communication

24. Harry J. Lipkin, in Proceedings of the Rheinfels Wcrkshop 1990 on Hadron Mass Spectrum, St.Goar at the Rhine, Germany, Sepi. 3-6, 1990, Nucl. Phys. B(Proc. Suppl.) 21 (1991) 258

25. S. May-Tal Beck, (for FNAL E791 Collab.) In Proceedings of the 1994 Annual Meeting of the Division of Particles and Fields, Albuquerque, N.M., S. Seidel, Ed., World Scientific, (1995) 1177

26. E.M. Aitala et al., FERMILAB-Pub-97/118-E and to be published

27. Daniel Ashery, These Proceedings

28. M. A. Moinester, D. Ashery, L. G. Landsberg and H. J. Lipkin, Zeitschrift fur Physik A 356 (1996) 207 